\title{
Quo vadis non-targeted wine analysis?
}

\author{
C. Fauhl-Hassek ${ }^{\mathrm{a}}$ \\ German Institut for Risk Assessement, Department Safety in the Food Chain, Max-Dohrn-Str. 8-10, 10589 Berlin, Germany
}

\begin{abstract}
Important challenges of wine authentication - for example the proof of wine varieties, the determination of the vintage or the geographical origin, the determination of certain practices (e.g. differentiation of barrel/chips storage) become more and more successfully investigated by non-targeted analytical approaches. Not only in relation to these open questions in wine authentication non-targeted approaches such as nuclear magnetic resonance and mass spectrometry offer enormous potential also in wine authentication due to their typical abilities as high-through put and screening technique.

The adaptation from research into routine applications takes actually place in some instances (e.g. NMR) including wine analysis. In addition the combination of non-targeted and targeted approaches within the same run of analysis provides possibilities complementing or even substituting classical methodologies.

Some examples, the general workflow and typical evaluation strategies will be presented and their possibilities and limits discussed. First results of a recent study on the NMR analysis with focus on variety verification and reproducibility of spectral data will be presented. Particular emphasis will be put on general open questions such as exchangeability of data, validation, standardisation options.
\end{abstract}

\section{NMR-spectroscopy}

The ${ }^{1} \mathrm{H}-\mathrm{NMR}$ analysis of aqueous matrices, such as wine [1], is especially challenging due to the dominant water signal as well as ethanol signals in the fingerprints masking interesting information. The acquisition of data has made tremendous progress during the last decade. Signal suppression of water, and more recently other major solvents has become routine (e.g. ethanol) [2] and the repeatability and reproducibility are improved [3], setting the prerequisite for databanks of spectral information.

Identification of different key wine ingredients and their quantification is possible by ${ }^{1} \mathrm{H}-\mathrm{NMR}$ spectroscopy in a few minutes. The sample preparation for wine analysis by NMR is straight forward, in principle only the addition of deuterated water is required. The NMR signals of certain substances in wine e.g. organic acids show dispersion in their chemical shifts according to the $\mathrm{pH}$ value of the sample. Therefore preparation protocols for wines often include the thorough adjustment of the $\mathrm{pH}$ in the samples [4], in order to keep the chemical shifts of the signals constant. Also the sample temperature needs to be controlled and kept very precisely to minimize chemical shift dispersion. Under quantitative measurement conditions every proton in the NMR tube contributes to same amount to the signal, whereas the shift depends on the environment (inter and intra molecular) of the proton. In order to quantify ingredients appropriate signal assignment as well as the reference measurement ether by simple internal standard addition or by external calibration, the so called PULCON procedure [5].

Figure 1 shows a typical ${ }^{1} \mathrm{H}-\mathrm{NMR}$ spectrum of a white wine, which was acquired with water and ethanol suppression. Several signals are assigned to their relevant

\footnotetext{
a e-mail: carsten.fauhl-hassek@bfr.bund.de
}

ingredient $(1=$ acetic acid, $2=$ succinic acid, $3=$ malic acid, $4=$ methanol, $5=$ glucose, malic acid, $6=$ tartaric acid).

Furthermore to the quantification of ingredients and the detection of known adulterants, the ability to detect abnormalities such as unknown adulterants, and the assessment of challenging authentication parameters (grape variety, origin, vintage) are possible by NMR spectroscopy [1].

The application of NMR spectroscopy for non-targeted analysis includes several processing steps [6]. For the multivariate data evaluation typically procedures for binning/bucketing are performed. After data acquisition a procedure that is called binning or bucketing is often performed, whereby small chemical shift regions (bins or buckets, e.g. $0.1 \mathrm{ppm}$ ) are summed together into one new one. Thus, the number of variables is reduced and small shifts are equalised. The data matrix then is the input for further multivariate statistical evaluations. Typically so called un-supervised methods such as Principle Component Analysis (PCA) are applied to discover data structure e.g. grouping according to variety or origin, on the other hand supervised methods such as Discriminant Analysis(DA) for classification questions.

Very important but not fully established yet in nontargeted analysis is use of quality assurance samples. Figure 2 shows an example how the quality of such a workflow including multivariate data evaluation could be assessed. Essential here is that the spread of the repeated sample measurement is lower than the natural variation of the group of reference samples.

As result of the mentioned advantages and technical progress practical applications using ${ }^{1} \mathrm{H}-\mathrm{NMR}$ spectroscopy $(400 \mathrm{MHz})$ in the field of food analysis and authentication were developed and are commercially 


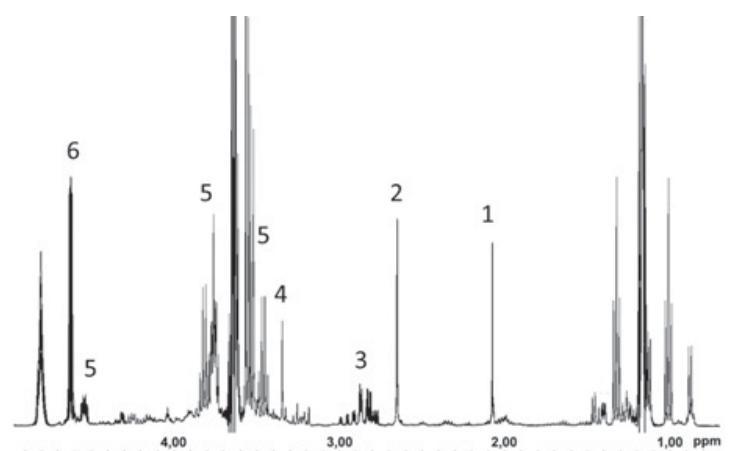

Figure 1. 1H-NMR spectrum of white wine, water and ethanol signals suppressed.

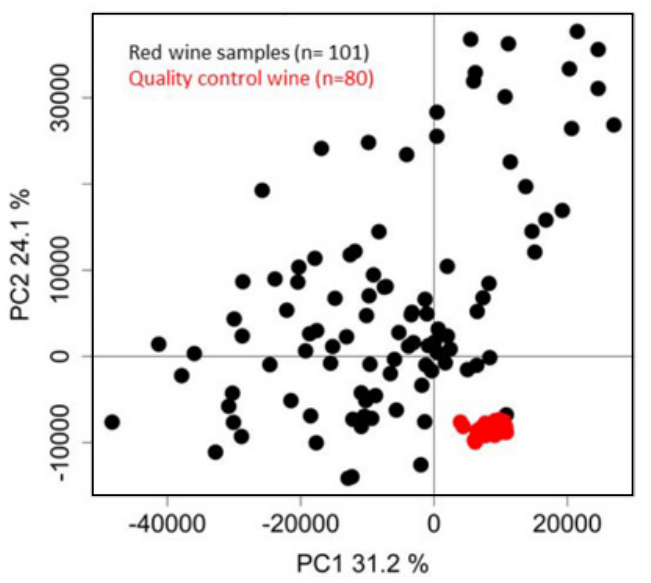

Figure 2. PCA of red wines samples and quality control wine.

available by the Bruker Corporation. The methodology aims to combine the utility of quantitative NMR (qNMR) dedicated to the quantification of wine ingredients with non-targeted analysis using the comparison with extensive reference databases for authentication purposes. The aim of the classification analysis in the case of the WineScreener ${ }^{\mathrm{TM}}$ for example is the verification of the grape variety, geographical origin and vintage. This is a stepwise process and includes several statistical models which are set-up in a decision tree/cascade.

\section{Mass spectrometry}

Mass spectrometry used for non-targeted wine analysis is linked to higher efforts in terms of data preparation because when coupled to chromatography 3-dimensional data are produced which are not or only by some sophisticated algorithms accessible for further evaluation.

In most of the studies chromatographic separation was performed employing gas chromatography [7] or liquid chromatography [8] before the mass spectrometric analysis. Particularly high resolution mass spectrometry combined with multivariate data analysis, PCA and DA or specific modifications, was demonstrated to be a potential tool for wine authentication in terms of grape varieties. Analysis of hundreds of samples each with thousands of signals (features) used for statistical analysis may take weeks, which can result in variable instrumental response or chemical changes, such as oxidation of wine samples. Particularly for mass spectrometric nontargeted approaches with high sensitivity the stability and
Chromatography hyphenated with mass spectrometry
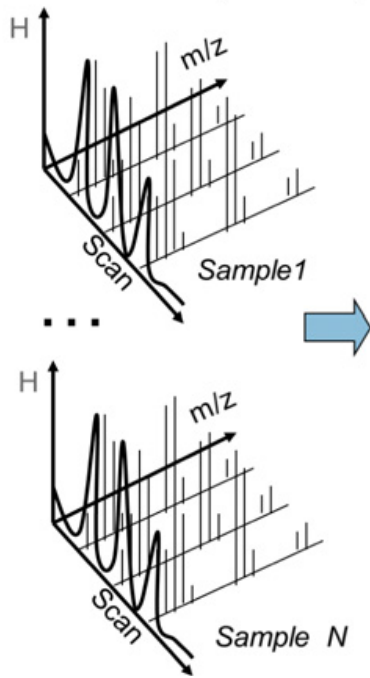

data preprocessing

$\mathrm{m} / \mathrm{z}$ _Scan

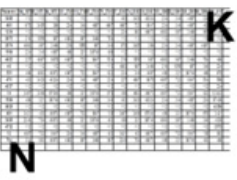

Sample

Figure 3. De-folding of 3-D data by combining $\mathrm{m} / \mathrm{z}$ and retention time (scan number).

consistency of models obtained over longer time as well using different instruments needs to be developed in order to confirm the validity of the models and to promote its application in routine. Often the time period in which the samples have been analysed is not stated, although this is a very important factor for assessing the model quality and stability.

Apart from its higher sensitivity one major advantage of mass spectrometry is the beneficiary identification of marker substances - compared to NMR spectroscopy for certain properties, for example varieties. When markers for certain varieties are identified and confirmed unequivocally, simpler analytical methods could be applied to provide control authorities with applicable routine methods. However, this was not published so far.

\section{Conclusion}

Although very interesting results are obtained and have been presented so far, typically feasibility or research studies are undertaken within one laboratory on one instrument exclusively, which restricts validation possibilities, particularly concerning the non-targeted approaches. Validation of the whole analytical procedure including statistical data evaluation and consistency of the measurement over time, instruments and laboratories is, however, essential for routine application and in official control. Therefore the use of non-targeted fingerprinting approaches is limited for official control purposes, due to actual missing validation strategies, thereby offering complex challenges for the scientific community.

\section{Literature}

[1] M. Amargianitaki, A. Spyros, Chem. Biol. Tech. Agr. 4, 9 (2017)

[2] Y.B. Monakhova, H. Schäfer, E. Humpfer, M. Spraul, T. Kuballa, D.W. Lachenmeier, Magn. Reson. Chem. 49, 734 (2011)

[3] A.P. Minoja, C. Napoli, Food Res. Int. 63, 126 (2014)

[4] S. Esslinger, C. Fauhl-Hassek, R. Wittkowski, Acs. Sym. Ser. 1203, 85 (2015) 
[5] G. Wider, L. Dreier, J. Am. Chem. Soc. 128, 2571 (2006)

[6] R. Godelmann, F. Fang, E. Humpfer, B. Schütz, M. Bansbach, H. Schäfer, M. Spraul, J. Agric. Food Chem. 61, 5610 (2013)
[7] Springer, T. Roth, M. Glomb, R. Wittkowski, C. Fauhl-Hassek, J. Agr. Food Chem. 62, 6844 (2014)

[8] J. Rubert, O. Lacina, C. Fauhl-Hassek, J. Hajslova, Anal. Bioanalytical Chem. 406, 6791 (2014) 